\title{
Letter to Editor
}

\section{Surgical Value of Neurocysticercosis}

\author{
Beuy JOOB ${ }^{1}$, Viroj WIWANITKIT ${ }^{2}$ \\ 'Sanitation 1 Medical Academic Center, Medical Center, Bangkok, Thailand \\ ${ }^{2}$ Hainan Medical University, Department of Tropical Medicine, Haikou, China
}

Dear Editor,

$\mathrm{T}$ The recent article on surgical value of neurocysticercosis (NCC) is very interesting (3). Xiao et al.(3) concluded that "As for extraparenchymal NCC, surgical therapy is a valuable treatment as an alternative due to its fatal complications" and "For the patient with intraventricular NCC associated with hydrocephalus, the effect of a simple VP shunt seems unsatisfactory, and craniotomy for cyst resection is necessary".

We would like to share our ideas and experience on this topic. The first issue is whether surgical removal is required or not for NCC. Some NCC might not require surgical treatment or surgery may be contraindicated and the use of conservative treatment is an interesting choice. In the report by Chotmongkol (2), the author stated that a 2-week course of albendazole is effective for the treatment of NCC. Another issue is the complications of surgery. Major surgery might result in post-surgical complications.
The use of a minimally invasive surgical technique such as endoscopic surgery is the current interesting alternative surgical approach for the management of NCC (1).

\section{REFERENCES}

1. Canaval Zuleta HJ, Company Campins MM, Dolz Abadía C: Endoscopy as a diagnostic and therapeutic alternative technique of taeniasis. Rev Esp Enferm Dig 108:371-376, 2016

2. Chotmongkol V: Treatment of neurocysticercosis with a two week course of albendazole. Southeast Asian J Trop Med Public Health 24:396-398, 1993

3. Xiao A, Xiao J, Zhang X, You C: Surgical value of neurocysticercosis: Analyzing 10 patients in 5 years. Turk Neurosurg 26:744-749, 2016 